\title{
Reward Preferences of Pathological Gamblers Under Conditions of Uncertainty: An Experimental Study
}

ARTICLE in JOURNAL OF GAMBLING STUDIES · FEBRUARY 2016

Impact Factor: 2.29 · DOI: 10.1007/s10899-016-9593-y

READS

61

5 AUTHORS, INCLUDING:

Maria Ciccarelli

Second University of Naples

3 PUBLICATIONS 0 CITATIONS

SEE PROFILE

Giovanna Nigro

Second University of Naples

35 PUBLICATIONS 159 CITATIONS

SEE PROFILE
Mark D Griffiths

Nottingham Trent University

754 PUBLICATIONS 15,067 CITATIONS

SEE PROFILE

Marina Cosenza

(4) Second University of Naples

17 PUBLICATIONS 33 CITATIONS

SEE PROFILE 


\title{
Reward preferences of pathological gamblers under conditions of uncertainty: An experimental study
}

\author{
Maria Ciccarelli ${ }^{1 *}$, Roberto Malinconico ${ }^{2}$, Mark D Griffiths. ${ }^{3}$, Giovanna Nigro ${ }^{1}$, \\ Marina Cosenza ${ }^{1}$ \\ 1 Department of Psychology, Second University of Naples, Caserta, Italy \\ 2 Department of Addictions, Local Health Trust, Caserta, Italy \\ 3 Department of Psychology, Nottingham Trent University, UK \\ *Corresponding author. e-mail: maria.ciccarelli@unina2.it; telephone number: 3331571608
}

\begin{abstract}
Among many personality traits, impulsivity represents one of the most important traits associated with pathological gambling. Empirical research has highlighted the multidimensional nature of impulsivity, which includes different heterogeneous traits and behavioral tendencies. The present study experimentally examined reward preferences of pathological gamblers under conditions of uncertainty using the Balloon Analogue Risk Task. Furthermore it also examined the relationship between impulsivity, time perspective, inability to tolerate delay in gratification, and risktaking. The present study is the first to simultaneously examine all these variables simultaneously in a sample of pathological gamblers $(n=54)$ and healthy controls $(n=54)$ from Italy. All participants participated in the Balloon Analogue Risk Task (BART) and were also administered Italian versions of the South Oaks Gambling Screen, the Barratt Impulsiveness Scale, the Consideration of Future Consequences, and the Monetary Choice Questionnaire. Analyses revealed that compared to HCs, PGs were more risk prone on the BART, and reported elevated levels of impulsivity, steeper discounting rates and a shorter time perspective. All the measures correlated
\end{abstract}


with the gambling severity and strong correlations between the BIS, CFC-14 and BART were observed. Logistic regression analysis demonstrated that impulsivity and risk-taking were strong predictors of pathological gambling.

Keywords: pathological gambling, risk-taking, impulsivity, time perspective, delay discounting. 


\section{Introduction}

Gambling is a form of socially accepted entertainment that for a small percentage of the population represents a serious problem of mental health that can negatively impact on the quality of life for people that experience it (Kessler, Hwang, LaBrie, Petukhova, Sampson, Winters, \& Shaffer, 2008;

Lorains, Cowlishaw, \& Thomas, 2011), leading to detrimental consequences such as job loss, bankruptcy, suicide, divorce, impaired relationships, and other health-problems (Lesieur \& Rosenthal, 1991; Griffiths, 2004). The degree of involvement in gambling lies on a continuum including problematic through to pathological, based on the number of symptoms that satisfy DSM criteria. Furthermore, it is not uncommon to find comorbidity between gambling and other potentially addictive behaviors such as smoking cigarettes, drinking alcohol, and illicit drug use (e.g., Griffiths, Wardle, Orford, Sproston, \& Erens, 2010; Ramirez, McCormick, Russo, \& Taber, 1983).

Recently, the large body of research has led to a change in the diagnostic classification of gambling (Potenza, 2006), from an impulse control disorder (American Psychiatric Association, 1980) to a behavioral addiction (American Psychiatric Association, 2013). Gambling disorder has become a psychopathological condition of dependence different to others in that it does not involve the ingestion of a psychoactive substance that cause harmful effects in the brain (Bechara, 2003; Potenza, 2001). This reclassification is due to the many characteristics that gambling shares with 
substance abuse (Griffiths, 2005). Both gambling and substance abuse disorders show manifestations of craving (Tavares, Zilberman, Hodgins, \& El-Guebaly, 2005), withdrawal (Griffiths \& Smeaton, 2002; Wray \& Dickerson, 1981) and tolerance (Griffiths, 1993), and an etiological core that includes genetic vulnerability (Slutske, Eisen, True, Lyons, Goldberg, \& Tsuang, 2000), and personality traits that predict the development of both the disorders (Vitaro, Arseneault, \& Tremblay 1999; Slutske, Caspi, Moffitt, \& Poulton, 2005).

Among many personality traits, impulsivity represents one of the most important traits associated with problem gambling (Alessi \& Petry, 2003; Cyders \& Smith, 2008; MacKillop, Anderson, Castelda, Mattson, \& Donovick, 2006a). The previous diagnostic classification of problem gambling as an Impulse Control Disorder (American Psychiatric Association, 1980) was arguably well founded as many studies have demonstrated the role of impulsivity in gambling and numerous studies have revealed significant differences in impulsivity between individuals with problem gambling compared to control groups (e.g., Canale, Vieno, Griffiths, Rubaltelli, \& Santinello, 2015; MacKillop, Anderson, Castelda, Mattson, \& Donovick, 2006b; Michalczuk, Bowden-Jones, Verdejo-Garcia, \& Clark, 2011).

A large body of research has brought to light the multidimensional nature of impulsivity, which includes different heterogeneous traits and behavioral tendencies (Canale et al., 2015; Flory, Harvey, Mitropoulou, New, 
Silverman, Siever, \& Manuck, 2006; Reynolds, Ortengren, Richards, \& de Wit, 2006; Swann, Bjork, Moeller, \& Dougherty, 2002; Whiteside \& Lynam, 2003). This paper focuses on facets of impulsivity that specifically relate to monetary choices, and which are particularly relevant to gambling addiction, given the nature of the disorder, in regards to risk-taking and delay discounting.

Delay discounting is the process of devaluing outcomes that require waiting (see Green \& Myerson [2004] for a review), and refers to the propensity to discount steeper conspicuous delayed rewards in favor of smaller immediate ones (Bickel \& Marsch, 2001; Rachlin \& Green, 1972). In the field of gambling, there is a considerable evidence that problem gamblers discount delayed money at higher rates than non-problem gamblers or non-gamblers (e.g., Alessi \& Petry, 2003; Brevers et al., 2012; Kräplin et al., 2014; Miedl, Peters, \& Büchel, 2012; Petry \& Casarella, 1999; Petry, 2012). Previous studies indicated that risk proneness is associated with problem gambling: gamblers tend to be more risk prone than non-gamblers and the level of monetary risk-taking appears to be exacerbated by continued exposure to gambling activities (Ladouceur, Mayrand, \& Tourigny, 1987; see also Martins, Tavares, da Silva Lobo, Galetti, \& Gentil, 2004; Petry, 2001b). The choice of gamble is a risk-taking behavior, based on the consideration of the immediate benefits that stem from the game, without considering the consequences that it may cause in the long-term, such as job loss, relationship and family problems or bankruptcy. Risk taking and delay 
discounting appear to be associated with the tendency to ignore the future consequences of actual behavior (Whiteside, Lynam, Miller, \& Reynolds, 2005). Strathman and colleagues defined the consideration of future consequences as "the extent to which people consider the potential distant outcomes of their current behaviors and the extent to which they are influenced by these potential outcomes" (Strathman, Gleicher, Boninger, \& Edwards, 1994; p. 743).

Despite data in support of the higher level of impulsiveness (see MacKillop et al. [2014] for a review) and the inability to tolerate delay in gambling, few studies have tested individuals with a diagnosis of gambling disorder according to DSM criteria (see Wiehler \& Peters, [2015] for a review) and the main limitation of these studies has been the use of small samples (e.g., Dixon, Marley, \& Jacobs, 2003, Dixon, Jacobs, \& Sanders, 2006; Miedl, Peters, \& Büchel, 2012; Miedl, Büchel, \& Peters, 2014; Kraplin et al., 2014). Likewise, few studies have dealt with the time perspective (Hodgins \& Engel, 2002) and with the propensity of gamblers to take risks (Ladouceur, Mayrand, \& Tourigny, 1987). In addition, no study has ever examined risk proneness in individuals with a diagnosis of problem gambling with a behavioural measure such as the Balloon Analogue Risk Task that simulates real-world risky situations in which excessive risk increases the amount of reward that may be won but where greater risk results in a higher likelihood of loss. 
In addition, some issues remain unanswered. Although several authors consider delay discounting as a behavioral feature of impulsivity (e.g., Ainslie, 1975; de Wit, 2009), the literature has not clarified the nature of this relationship. Some studies have observed an association between the two constructs (e.g., Alessi \& Petry, 2003; Andrade \& Petry, 2012; Kräplin et al., 2014; Petry, 2001a, 2012), whereas other studies have not confirmed this pattern of results (e.g., Madden, Petry, \& Johnson, 2009; Wilde, Goudriaan, Sabbe, Hulstijn, \& Dom, 2013).

Further, albeit the association between impulsivity and time perspective has been observed in adolescents (Cosenza \& Nigro, 2015) and adult problem gamblers (MacLaren, Fugelsang, Harrigan, \& Dixon, 2012; Toplak, Liu, MacPherson, Toneatto, \& Stanovich, 2007), no study has ever examined this relationship in a sample of pathological gamblers. Moreover, there is no agreement about the association between the inability to tolerate delay in gratification and addiction severity (Alessi \& Petry, 2003; Brevers et al., 2012; Kräplin et al., 2014; Madden et al., 2009; Miedl et al., 2012; Petry, 2001a, 2012).

Given these premises, the purpose of this study was to investigate, for the first time, all together, the role of impulsivity, time perspective, delay discounting, and risk-taking in a sample of pathological gamblers and healthy controls.

It was hypothesized that pathological gamblers, compared to nonpathological gamblers, would be more risk prone using the Balloon 
Analogue Risk Task. It was also hypothesized that, compared to healthy controls, pathological gamblers would be more impulsive, more oriented to the present, and report a steeper delay discounting. It was further hypothesized that there would be high correlations between these different features of impulsivity and pathological gambling.

\section{Method}

\section{Participants}

The sample comprised 108 Italian male volunteers with an age range of 2465 years $($ mean $=41.56$ years; $\mathrm{SD}=10.94)$. Of these, 54 were pathological gamblers (PGs) recruited from the Local Health Trust (in the Italian cities of Caserta, Salerno and Naples). None of the participants had comorbid Axis-I or II disorders, alcohol or substance abuse, neurological or medical illnesses, or were undergoing any pharmacological treatment. It should also be noted that the pathological gamblers were recruited from the Local Health Trust and were diagnosed using the DSM-5 criteria (American Psychiatric Association, 2013). Gamblers who met the criteria for pathological gambling (i.e., endorsing 5 or more out of the 9 criteria) were eligible for participation in the study. These were matched with 54 healthy controls (HCs) recruited from the local community. The controls were selected randomly from a large sample of adults without pre-existing psychiatric/neurological disorder or problem gambling. There were no 
significant differences in mean age $\left(\mathrm{t}_{106}=-.48, p=0.63\right)$ or years of education $\left(\mathrm{t}_{106}=1.59, p=0.12\right)$ between the two groups.

\section{Measures}

Balloon Analogue Risk Task (BART; Lejuez, Read, Kahler, Richards, Ramsey, Stuart, \& Brown, 2002). The BART is a computerized measure of risky behavior that assesses reward preferences under conditions of uncertainty. It comprises a series of balloons that participant has to inflate by clicking a button on the screen. Each click inflates the balloon and accrues 5 cents in a temporary bank. The more the participant inflates the balloon, the more money accrues. After a certain unpredictable number of pumps, the balloon may burst causing the money accrued in the temporary bank to be lost. It is possible at any time for a participant to click on the button labeled "Collect $\$ \$ \$$ ", which transfers all money from the temporary bank to the permanent bank, where the money can no longer be lost, even if the next balloon bursts. The bursting of the balloon causes the loss of money in the temporary bank, while money in the permanent bank is safe.

The computer screen shows a balloon at the center alongside a series of buttons: "Collect $\$ \$ \$$ " transferring all money to a permanent bank, "Pump up the balloon" to pump the balloon and earn money, "Total Earned" showing all money accumulated in the permanent bank, and "Last Balloon", indicating money won with the last balloon (see Figure 1). The original version of the task comprises 90 trials with three different balloon 
types (i.e., blue, yellow, and orange), with each color having a different probability of explosion.

In the version used in the present study, there were 30 balloons in total, presented in a randomly computer-generated order to each participant. Participants did not know at which point the balloons would burst. They were told that each balloon could explode at any time and that the balloon could burst after the first pump up to balloon covering the whole screen. Participants sat in front of the computer and are asked to wear headphones. Risk-taking is assessed as the mean average number of pumps on un-popped balloons with higher scores indicate greater risk-taking (Lejuez et al., 2002).

South Oaks Gambling Screen (SOGS; Lesieur \& Blume [1987]; Italian translation by Cosenza, Matarazzo, Baldassarre, \& Nigro [2014]). The SOGS is a 20-item self-report instrument where each response is dichotomous (yes/no) and assesses the frequency and the gravity of gambling problems. Items include "When you gamble, how often you go back another day to win back the money you lost?" and "Have people ever criticized your gambling?" The items are based on the DSM criteria for problem gambling (American Psychiatric Association, 1980). Individuals who score from 0-2 are classified as having no problems with gambling, whereas those who score from 3-4 are classified as at-risk gamblers, and those with a score of 5 or above are classified as problem gamblers. The 
SOGS in the present study was found to have a high internal consistency reliability coefficient (Cronbach's alpha $=.93,95 \%$ CI $[.92, .94])$.

Barratt Impulsiveness Scale (BIS-11; Patton, Stanford, \& Barratt [1995]; Italian BIS-11 validation by Fossati, Di Ceglie, Acquarini, \& Barratt [2001]). The BIS-11 is a 30-item self-report questionnaire assessing trait impulsiveness in general, and three its dimensions in particular: attentional impulsivity, that is the inability to concentrate attention (e.g., "I am restless at the theater or lectures"), motor impulsivity, namely acting without thinking (e.g., "I act on the spur of the moment"), and nonplanning impulsivity, that is the lack of future thinking (e.g., "I am more interested in the present than the future"). Responses are provided using a 4-point Likert scale ranging from "rarely/never" to "almost always/always". Higher scores on the instrument indicate higher levels of impulsivity. In the present study, internal consistency for the total scale $(\alpha=.79,95 \%$ CI $[.73, .84])$ and for each scale was adequate in the present sample: attentional $(\alpha=.63,95 \% \mathrm{CI}$ $[.51, .72])$, motor $(\alpha=.72,95 \%$ CI $[.63, .79])$, and nonplanning $(\alpha=.60,95 \%$ CI $[.47, .70])$.

Consideration of Future Consequences (CFC-14; Strathman, Gleicher, Boninger, \& Edwards, 1994; Italian translation by Cosenza \& Nigro, 2015). The CFC-14 is a valid and reliable self-report questionnaire that assesses the extent to which individuals focus on the future consequences of their current 
behavior (i.e., it assesses time perspective). The 14 items comprise two subscales: immediate (e.g., "I generally ignore warnings about possible future problems because I think the problems will be resolved before they reach crisis level") and future (e.g., "I consider how things might be in the future, and try to influence those things with my day to day behaviour"), scored using a 7-point scale (from "extremely uncharacteristic" to "extremely characteristic"). Higher scores on the instrument indicate a greater tendency to consider future consequences. In the present study, Cronbach's alpha for the full scale was .75 (95\% CI $[.68, .82])$ and .74, $(95 \% \mathrm{CI}[.66, .81])$ for the immediate subscale, and $.80(95 \% \mathrm{CI}[.73, .85])$ for the future subscale.

Monetary Choice Questionnaire (MCQ; Kirby \& Maraković, 1996; Italian translation by Cosenza \& Nigro, 2015). MCQ is a questionnaire that assesses delay discounting and comprises 27 questions (using a dichotomous 'yes/no' scale), each of which involves a choice between an immediate amount of money and a larger amount delivered after a delay (ranging from 7 to 186 days). For example, one question asks, "Would you prefer $\$ 14$ today, or $\$ 25$ in 19 days?” Depending on the type of reward, questions are divided into three categories (i.e., small, medium and large). The pattern of responses determines the degree to which participants discount delayed rewards, by estimating $k$ values obtained at three magnitudes of rewards contained within the questionnaire (i.e., small, 
medium and large). Therefore, the items assess how much the amount of a postponed reward influences its perceived value. Following the example by Kirby \& Marakovic (1996) and Kirby, Petry, \& Bickel (1999), question 19 required participants to choose between "\$33 today" and "\$80 in 14 days." (in our version Euros). A discount rate of 0.10 indicates that the participant is indifferent between these two rewards. Therefore, if a participant chooses the immediate reward on this trial ("\$33”), his discount rate is greater than 0.10 . Question 4 required participant to choose between "\$31 today" and "\$85 in 7 days.". A discount rate of 0.25 indicates that the participant is indifferent between these two rewards. Therefore, if the same participant chooses the delayed reward on this trial, his discount rate is less than 0.25 . The two trials together imply that this person has a discount rate between 0.10 and 0.25 . The geometric mean of this interval $(k=0.16)$ is used to estimate the person's $k$ value. This procedure minimizes the number of choices that would be errors with respect to the assigned $k$ value (Kirby, 2009; p. 459). Higher $\mathrm{k}$ values reflect a preference for immediate rewards. In the present study, the monetary amounts were converted from U.S. dollars to Euros. Kuder-Richardson reliability for the total scale (KR-20= .89) and for each scale was adequate in the present sample: small k (KR-20= $.70)$, medium $\mathrm{k}(\mathrm{KR}-20=.71)$, and large $\mathrm{k}(\mathrm{KR}-20=.73)$.

\section{Procedure}


The participants were told that they were participating in an anonymous study in which the associations between several factors and gambling behavior were being examined. Participants were tested individually in a quiet room in a single session. After obtaining signed informed consent, participants performed the computerized BART task. Following this, they completed the battery of questionnaires. At the end of the experimental session, in the debriefing, the real purpose of the research was revealed. Each participant was thanked for his participation without monetary rewards. They were also assured that data would be analyzed in aggregate form and that they could withdraw from the study whenever they wanted. To ensure the anonymity of the participants, there were all assigned a numeric code. Ethical approval for the study was granted by the research team's university ethics committee.

\section{Statistical analyses}

Data were analyzed with the IBM Statistical Package for the Social Sciences, version 20.0. The alpha significance level was set at .05. A univariate ANOVA with 'group' (problem gamblers vs. healthy controls) as the independent variable and the average number of pumps in the BART as the dependent variable was conducted with the purpose to testing risktaking differences between the two groups. Univariate analyses of variance (ANOVAs) with impulsivity (BIS-11) and time perspective (CFC-14) as the dependent variables and 'group' (problem 
gamblers vs. healthy controls) as independent factor were used to verify whether these measures differed significantly between groups. Multivariate analyses of variance (MANOVA) with the 'group' (problem gamblers vs. healthy controls) as independent variable and subscale scores of BIS-11 and CFC-14 as dependent variables was conducted.

In order to evaluate delay-discounting differences between HCs and PGs, a mixed (2 X 3) analysis of variance (ANOVA) with 'group' (problem gamblers vs. healthy controls) as the between-participants and the three different reward amount-based parameters (small, medium and large $k$ ) as the within-participants factor.

Zero-order correlations were calculated to examine the relationship between SOGS, BIS-11, CFC-14, and MCQ scores.

Finally, a logistic regression was run with 'group' (problem gamblers vs. healthy controls) as dependent variable and total scores of behavioral (BART, MCQ rates) and self-report measures (BIS-11, CFC-14) as predictors.

\section{Results}

The effects of risk taking propensity as a function of gambling behavior severity were examined using the BART computerized experimental task. Results indicated that the two groups differed in risk-taking behavior (Table 1). PGs were more risk prone compared to HCs (see Figure 2). 
PGs scored significantly higher than HCs on general impulsiveness (BIS11), and specifically on attentional impulsiveness, motor impulsiveness, and non-planning impulsiveness scales (Table 1).

The two groups differed on time perspective (CFC-14), with PGs reporting significantly high scores on its subscale: immediate, compared to HCs. Future subscale scores were no different between groups. Analysis of delay discounting rates revealed a main effect of group: pathological gamblers showed steeper delay discounting rates than nonpathological gamblers. No 'group X reward' amount interaction was detected, $\left.\mathrm{F}(2,105)=0.15 ; p=.86, \eta^{2}=.01\right)($ see Figure 3$)$. Correlational analysis (see Table 2) revealed associations between all of the measures and severity of gambling problems. Significant patterns of association were observed between impulsivity (BIS), risk proneness (BART), and orientation to the present (CFC-14). In contrast, delay discounting rate (average $k$ ) correlated only with the severity of gambling problems, whereas Medium $k$ rate was associated with motor impulsiveness and non-planning impulsiveness (see Table 2).

A logistic regression was also run in order to identify the predictor of severity of gambling problems. Total scores on the BIS-11 and CFC-14, the overall discounting rate (Average $k$ ), and the performance on the Balloon Analogue Risk Task were used as predictor variables. In the first step, impulsivity (BIS-11) significantly predicted problem gambling, $\chi^{2}(1, n=108)$ $=24.11, p<.001$, with $27 \%$ of variance explained (Nagelkerke $\left.\mathrm{R}^{2}\right)$. In the 
second step, along with impulsivity, risk-taking (BART) was entered into the regression model, $\chi^{2}(2, n=108)=29.53, p<.001$ and added $5 \%$ of the variance to the model. The betas indicated that impulsivity and risk-taking predict problem gambling. The percentage of explained variance in the full model was $32 \%$. The overall classification accuracy was $68 \%$. The Table 3 displays the standardized beta coefficients, Wald statistics and significance levels for the predictors included in the model.

\section{Discussion}

This study experimentally examined, for the first time, the difference between pathological gamblers and healthy controls in being risk prone on the Balloon Analogue Risk Task (BART), a validated task that resembles the real-world risk (Lejuez et al., 2002). The study also investigated, all together, impulsivity, inability to tolerate delay in gratification and time orientation in a sample of pathological gamblers and healthy controls, exploring the links between these factors and pathological gambling. The results showed that, compared to healthy controls (HCs), pathological gamblers were more risk prone and reported higher scores than $\mathrm{HCs}$ on all measures tested (i.e., increased impulsivity, steeper discounting rates, more orientation to the present). Analyses revealed significantly strong correlations between impulsivity (BIS-11), orientation to the present (CFC14), and risk proneness (BART), whereas delay discounting (MCQ) was only associated with the severity of gambling problems. Moreover, all the 
measures were related to gambling severity. Finally, logistic regression analysis demonstrated that impulsivity, along with risk-taking, was a significant predictor of gambling severity.

Pathological gamblers were found to pump up balloons significantly more times than controls, thus demonstrating high-risk proneness, in line with past research (e.g., Ladouceur et al., 1987). This result is interesting as regards not the generic risk-taking but specifically monetary choices. In fact, even if the potential monetary wins or losses in the Balloon Analogue Risk Task were fictitious, participants were asked to behave as if money were real. In literature, the validity of the BART in capturing risk-taking has been well demonstrated (e.g., Lejuez et al., 2002).

In contrast with Reynolds et al. (2006), a relationship between impulsivity and risk-taking was observed. The contradictory nature of results of these two studies is probably due to the heterogeneity of the two samples. An interesting association between time perspective and risk proneness was found, indicating that individuals with a shorter time perspective and difficulty considering future acting, were more likely to engage in risky behavior.

Consistent with previous research that, starting from the classification of gambling as "impulse control disorder" has revealed the strong relation between impulsivity and problem gambling, the present study confirmed that the level of impulsivity is higher in pathological gamblers compared to controls (e.g., MacKillop, Anderson, Castelda, Mattson, \& Donovick, 
2006b; Michalczuk, Bowden-Jones, Verdejo-Garcia, \& Clark, 2011). Even if, in this study, the direction of the relationship between impulsivity and problem gambling severity cannot be inferred, previous longitudinal studies suggest that impulsivity causes gambling (Auger, Lo, Cantinotti, \& O'Loughlin, 2010; Pagani, Derevensky, \& Japel, 2009; Shenassa, Paradis, Dolan, Wilhelm, \& Buka, 2012; Vitaro et al., 1999). For instance, Shenassa et al. (2012) found that children that exhibit higher level of impulsivity have a greater risk for developing gambling problems during adulthood. In line with previous findings (Hodgins \& Engel, 2002), pathological gamblers reported also a short temporal horizon, with orientation to the present and an inability to consider a long period of time into the future. The correlation between time perspective and the three dimensions of impulsivity (i.e., attentional, motor, and non-planning) indicated that individuals that are oriented to the present tend to act without thinking and tend to make rapid decisions without future planning. This result observed in pathological gamblers confirms and extends previous findings on convenience samples of undergraduate students (Baumann \& Odum, 2012; Daugherty \& Brase, 2010) and on problem gamblers screened with selfreport questionnaire (Toplak et al., 2007).

Similarly to previous studies (Alessi \& Petry, 2003; Dixon, Marley \& Jacobs, 2003; Miedl et al., 2012; Kraplin et al., 2014), the steeper discounting rates found in pathological gamblers, compared to controls, indicated that people with a pathological involvement in gambling prefer 
immediate rewards, even if smaller, than delayed ones, showing the inability to tolerate delay in gratification.

The association between the inability to tolerate delay and gambling severity, in contrast with past studies (Madden et al., 2009; Miedl et al., 2012; Petry, 2001a; Petry, 2012; Wilde, Goudriaan, Sabbe, Hulstijn, \& Dom, 2013) confirms the pattern observed in Alessi and Petry's study (2003). Even if this study does not permit a comprehensive insight into the nature of this relationship, recent research on problem and non-problem gambling adolescents highlights that delay discounting is one of the strongest predictor of gambling problems (Cosenza \& Nigro, 2015). Similarly, in substance abuse literature, several research studies have observed that abstinence is associated with a reduction of discounting rates (Bickel, Odum, \& Madden, 1999; Bretteville-Jensen, 1999; Petry, 2001a). The observed (albeit relatively weak) association between delay discounting, motor, and non-planning impulsiveness, confirmed past studies (e.g., Baumann \& Odum, 2012; Cosenza \& Nigro, 2015; de Wit, Flory, Acheson, McCloskey, \& Manuck, 2007) and indicates that delay discounting should probably be considered a feature of impulsivity. Finally, high impulsivity, shortened time perspective and risk-taking were correlated with the severity of gambling problems.

The present study is not without its limitations. The main limitation was the use of self-report measures that are influenced by many biases including recall biases and social desirability biases. The lack of associations between 
time perspective and delay discounting, that was expected, represents another limitation of this study. The sample size was also small but, compared to other studies with an experimental component, the sample size was commensurate. Also, the number of pathological gamblers in the study was relatively large compared to other studies. For instance, most national prevalence studies with sample sizes of 7.000-10.000 participants only contain 50-70 problem gamblers when analysis is done comparing problem gamblers to non-problem gamblers (e.g., the British Gambling Prevalence surveys by Wardle et al. [2007; 2011]). Despite the limitation of self-report, the results of the present study demonstrate correlations among several impulsivity traits in healthy and pathological gamblers, supporting the hypothesis that delay discounting, risk proneness and time perspective are not only related to each other but are important factors in gambling disorder. The present study also underlines that impulsivity is a core feature of gambling and that all its correlates need to be taken into account for a wider and more comprehensive understanding of this aspect of the disorder. In summary, the present study confirms many previous findings in the literature, and demonstrates that gamblers tend to be impulsive, oriented to the present and that they prefer immediate gratification and show more pronounced risk proneness. Future studies should investigate other psychological factors involved in pathological gambling that may be a role in impulsive behavior, such as motivational factors such as craving. 
It would also be interesting to establish if the effects found in the present study are observable in the early stage of the onset of gambling problems, including adolescent populations. 


\section{References}

Ainslie, G. (1975). Specious reward: a behavioral theory of impulsiveness and impulse control. Psychological Bulletin, 82, 463-496.

Alessi, S. M., \& Petry, N. M. (2003). Pathological gambling severity is associated with impulsivity in a delay discounting procedure. Behavioural Processes, 64, 345-354.

American Psychiatric Association. (2000). Diagnostic and Statistical Manual of Mental Disorders (4th ed. Text Revision). Washington, D.C.: American Psychiatric Association.

American Psychiatric Association (2013). Diagnostic and Statistical Manual of Mental Disorders (5th ed.). Arlington, VA: American Psychiatric Publishing.

Andrade, L. F., \& Petry, N. M. (2012). Delay and probability discounting in pathological gamblers with and without a history of substance use problems. Psychopharmacology, 219, 491-499.

Auger, N., Lo, E., Cantinotti, M., \& O'Loughlin, J. (2010). Impulsivity and socio-economic status interact to increase the risk of gambling onset among youth. Addiction, 105, 2176-2183.

Baumann, A. A., \& Odum, A. L. (2012). Impulsivity, risk taking, and timing. Behavioural Processes, 90, 408-414.

Bechara, A. (2003). Risky business: emotion, decision-making, and addiction. Journal of Gambling Studies, 19, 23-51. 
Bickel, W. K., \& Marsch, L. A. (2001). Toward a behavioral economic understanding of drug dependence: delay discounting processes. Addiction, 96, 73-86.

Bickel, W. K., Odum, A. L., \& Madden, G. J. (1999). Impulsivity and cigarette smoking: delay discounting in current, never, and ex-smokers. Psychopharmacology, 146, 447-454.

Bretteville-Jensen, A. L. (1999). Addiction and discounting. Journal of Health Economics, 18, 393-407.

Brevers, D., Cleeremans, A., Verbruggen, F., Bechara, A., Kornreich, C., Verbanck, P., \& Noël, X. (2012). Impulsive action but not impulsive choice determines problem gambling severity. PLoS ONE, 7(11): e50647. doi:10.1371/journal.pone.0050647

Canale, N., Vieno, A., Griffiths, M.D., Rubaltelli, E., Santinello, M. (2015). How $\underline{\text { do impulsivity traits influence problem gambling through gambling }}$ motives? The role of perceived gambling risk/benefits. Psychology of Addictive Behaviors 29, 813-823.

Cosenza, M., Matarazzo, O., Baldassarre, I., \& Nigro, G. (2014). Deciding with (or without) the Future in Mind: Individual Differences in Decision-Making. InRecent Advances of Neural Network Models and Applications (pp. 435443). Springer International Publishing.

Cosenza, M., \& Nigro, G. (2015). Wagering the future: Cognitive distortions, impulsivity, delay discounting, and time perspective in adolescent gambling. Journal of Adolescence, 45, 56-66. 
Cyders, M. A., \& Smith, G. T. (2008). Emotion-based dispositions to rash action: Positive and negative urgency. Psychological Bulletin, 134, 807-846.

Daugherty, J. R., \& Brase, G. L. (2010). Taking time to be healthy: Predicting health behaviors with delay discounting and time perspective. Personality and Individual Differences, 48(2), 202-207.

de Wit, H. (2009). Impulsivity as a determinant and consequence of drug use: a review of underlying processes. Addiction Biology, 14, 22-31.

de Wit, H., Flory, J. D., Acheson, A., McCloskey, M., \& Manuck, S. B. (2007). IQ and nonplanning impulsivity are independently associated with delay discounting in middle-aged adults. Personality and Individual Differences, $42,111-121$.

Dixon, M. R., Jacobs, E. A., \& Sanders, S. (2006). Contextual control of delay discounting by pathological gamblers. Journal of Applied Behavior Analysis, 39, 413-422.

Dixon, M. R., Marley, J., \& Jacobs, E. A. (2003). Delay discounting by pathological gamblers. Journal of Applied Behavior Analysis, 36(4), 449458.

Flory, J. D., Harvey, P. D., Mitropoulou, V., New, A. S., Silverman, J. M., Siever, L. J., \& Manuck, S. B. (2006). Dispositional impulsivity in normal and abnormal samples. Journal of Psychiatric Research, 40, 438-447.

Fossati, A., Di Ceglie, A., Acquarini, E., \& Barratt, E. S. (2001). Psychometric properties of an Italian version of the Barratt Impulsiveness Scale-11 
(BIS-11) in nonclinical subjects. Journal of Clinical Psychology, 57, 815828.

Green, L., \& Myerson, J. (2004). A discounting framework for choice with delayed and probabilistic rewards. Psychological Bulletin, 130, 769-816.

Griffiths, M. (1993). Tolerance in gambling: An objective measure using the psychophysiological analysis of male fruit machine gamblers. Addictive Behaviors, 18, 365-372.

Griffiths, M. D. (2004). Betting your life on it: Problem gambling has clear health related consequences. British Medical Journal, 329, 1055-1056.

Griffiths, M. D. (2005). A ‘components' model of addiction within a biopsychosocial framework. Journal of Substance Use, 10, 191-197.

Griffiths, M. D. \& Smeaton, M. (2002). Withdrawal in pathological gamblers: A small qualitative study. Social Psychology Review, 4, 4-13.

Griffiths, M. D., Wardle, J., Orford, J., Sproston, K. \& Erens, B. (2010). Gambling, alcohol consumption, cigarette smoking and health: findings from the 2007 British Gambling Prevalence Survey. Addiction Research and Theory, 18, 208-223.

Hodgins, D. C., \& Engel, A. (2002). Future time perspective in pathological gamblers. Journal of Nervous and Mental Disease, 190, 775-780.

Kessler, R. C., Hwang, I., LaBrie, R., Petukhova, M., Sampson, N. A., Winters, K. C., \& Shaffer, H. J. (2008). DSM-IV pathological gambling in the National Comorbidity Survey replication. Psychological Medicine, 38, $1351-1360$. 
Kirby, K. N. (2009). One-year temporal stability of delay-discount rates.

Psychonomic bulletin \& review, 16(3), 457-462.

Kirby, K. N., \& Maraković, N. N. (1996). Delay-discounting probabilistic rewards: Rates decrease as amounts increase. Psychonomic Bulletin and Review, 3, 100-104.

Kirby, K. N., Petry, N. M., \& Bickel, W. K. (1999). Heroin addicts have higher discount rates for delayed rewards than non-drug-using controls. Journal of Experimental Psychology: General, 128(1), 78-87.

Kräplin, A., Dshemuchadse, M., Behrendt, S., Scherbaum, S., Goschke, T., \& Bühringer, G. (2014). Dysfunctional decision-making in pathological gambling: pattern specificity and the role of impulsivity. Psychiatry Research, 215, 675-682.

Ladouceur, R., Mayrand, M., \& Tourigny, Y. (1987). Risk-taking behavior in gamblers and non-gamblers during prolonged exposure. Journal of Gambling Behavior, 3, 115-122.

Lejuez, C. W., Read, J. P., Kahler, C. W., Richards, J. B., Ramsey, S. E., Stuart, G. L., ... \& Brown, R. A. (2002). Evaluation of a behavioral measure of risk taking: the Balloon Analogue Risk Task (BART). Journal of Experimental Psychology: Applied, 8(2), 75-84.

Lesieur, H. R., \& Blume, S. B. (1987). The South Oaks Gambling Screen (SOGS): a new instrument for the identification of pathological gamblers. American Journal of Psychiatry, 144, 1184-1188. 
Lesieur, H. R., \& Rosenthal, R. J. (1991). Pathological gambling: A review of the literature (prepared for the American Psychiatric Association task force on DSM-IV committee on disorders of impulse control not elsewhere classified). Journal of Gambling Studies, 7, 5-39.

Lorains, F. K., Cowlishaw, S., \& Thomas, S. A. (2011). Prevalence of comorbid disorders in problem and pathological gambling: systematic review and meta-analysis of population surveys. Addiction, 106, 490-498.

MacKillop, J., Anderson, E. J., Castelda, B. A., Mattson, R. E., \& Donovick, P. J. (2006a). Convergent validity of measures of cognitive distortions, impulsivity, and time perspective with pathological gambling. Psychology of Addictive Behaviors, 20, 75.

MacKillop, J., Anderson, E. J., Castelda, B. A., Mattson, R. E., \& Donovick, P. J. (2006b). Divergent validity of measures of cognitive distortions, impulsivity, and time perspective in pathological gambling. Journal of Gambling Studies, 22, 339-354.

MacKillop, J., Miller, J. D., Fortune, E., Maples, J., Lance, C. E., Campbell, W. K., \& Goodie, A. S. (2014). Multidimensional examination of impulsivity in relation to disordered gambling. Experimental and Clinical Psychopharmacology, 22, 176.

MacLaren, V. V., Fugelsang, J. A., Harrigan, K. A., \& Dixon, M. J. (2012). Effects of impulsivity, reinforcement sensitivity, and cognitive style on pathological gambling symptoms among frequent slot machine players. Personality and Individual Differences, 52, 390-394. 
Madden, G. J., Petry, N. M., \& Johnson, P. S. (2009). Pathological gamblers discount probabilistic rewards less steeply than matched controls. Experimental and Clinical Psychopharmacology, 17, 283-297.

Martins, S. S., Tavares, H., da Silva Lobo, D. S., Galetti, A. M., \& Gentil, V. (2004). Pathological gambling, gender, and risk-taking behaviors. Addictive Behaviors, 29, 1231-1235.

Michalczuk, R., Bowden-Jones, H., Verdejo-Garcia, A., \& Clark, L. (2011). Impulsivity and cognitive distortions in pathological gamblers attending the UK National Problem Gambling Clinic: A preliminary report. Psychological Medicine, 41, 2625-2635.

Miedl, S. F., Büchel, C., \& Peters, J. (2014). Cue-induced craving increases $\underline{\text { impulsivity via changes in striatal value signals in problem }}$ gamblers. Journal of Neuroscience, 34, 4750-4755.

Miedl, S. F., Peters, J., \& Büchel, C. (2012). Altered neural reward representations in pathological gamblers revealed by delay and probability discounting. Archives of General Psychiatry, 69, 177-186.

Pagani, L. S., Derevensky, J. L., \& Japel, C. (2009). Predicting gambling behavior in sixth grade from kindergarten impulsivity: a tale of developmental continuity. Archives of pediatrics \& adolescent medicine, 163, 238-243.

Patton, J. H., Stanford, M. S., \& Barratt, E. S. (1995). Factor structure of the Barratt impulsiveness scale. Journal of Clinical Psychology, 51, 768-74. 
Petry, N. M. (2012). Discounting of probabilistic rewards is associated with gambling abstinence in treatment-seeking pathological gamblers. Journal of Abnormal Psychology, 121, 151-167.

Petry, N. M. (2001a). Pathological gamblers, with and without substance abuse disorders, discount delayed rewards at high rates. Journal of Abnormal Psychology, 110, 482-487.

Petry, N. M. (2001b). Substance abuse, pathological gambling, and impulsiveness. Drug and Alcohol Dependence, 63, 29-38.

Petry, N. M., \& Casarella, T. (1999). Excessive discounting of delayed rewards in substance abusers with gambling problems. Drug and Alcohol Dependence, $\underline{56,25-32 .}$

Potenza, M. N. (2006). Should addictive disorders include non-substance-related conditions? Addiction, 101, 142-151.

Potenza, M. N. (2001). The neurobiology of pathological gambling. Seminars in Clinical Neuropsychiatry, 6, 217-226.

Rachlin, H., \& Green, L. (1972). Commitment, choice and self-control 1. Journal of the Experimental Analysis of Behavior, 17, 15-22.

Ramirez, L. F., McCormick, R. A., Russo, A. M., \& Taber, J. I. (1983). Patterns of substance abuse in pathological gamblers undergoing treatment. Addictive Behaviors, 8, 425-428.

Reynolds, B., Ortengren, A., Richards, J. B., \& de Wit, H. (2006). Dimensions of impulsive behavior: Personality and behavioral measures. Personality and Individual Differences, 40, 305-315. 
Shenassa, E. D., Paradis, A. D., Dolan, S. L., Wilhelm, C. S., \& Buka, S. L. (2012). Childhood impulsive behavior and problem gambling by adulthood: A 30-year prospective community-based study. Addiction, 107, 160-168.

Slutske, W. S., Caspi, A., Moffitt, T. E., \& Poulton, R. (2005). Personality and problem gambling: A prospective study of a birth cohort of young adults. Archives of General Psychiatry, 62, 769-775.

Slutske, W. S., Eisen, S., True, W. R., Lyons, M. J., Goldberg, J., \& Tsuang, M. (2000). Common genetic vulnerability for pathological gambling and alcohol dependence in men. Archives of General Psychiatry, 57, 666-673.

Strathman, A., Gleicher, F., Boninger, D. S., \& Edwards, C. S. (1994). The consideration of future consequences: Weighing immediate and distant outcomes of behavior. Journal of Personality and Social Psychology, 66, $742-752$.

Swann, A. C., Bjork, J. M., Moeller, F. G., \& Dougherty, D. M. (2002). Two models of impulsivity: relationship to personality traits and psychopathology. Biological Psychiatry, 51, 988-994.

Tavares, H., Zilberman, M. L., Hodgins, D. C., \& El-Guebaly, N. (2005). Comparison of craving between pathological gamblers and alcoholics. Alcoholism: Clinical and Experimental Research, 29, 1427-1431.

Toplak, M. E., Liu, E., MacPherson, R., Toneatto, T., \& Stanovich, K. E. (2007). The reasoning skills and thinking dispositions of problem gamblers: A dual-process taxonomy. Journal of Behavioral Decision Making, 20, 103124. 
Vitaro, F., Arseneault, L., \& Tremblay, R. E. (1999). Impulsivity predicts problem gambling in low SES adolescent males. Addiction, 94, 565-575.

Wardle, H., Sproston, K., Orford, J., Erens, B., Griffiths, M. D., Constantine, R. \& Pigott, S. (2007). The British Gambling Prevalence Survey 2007. London: The Stationery Office

Wardle, H., Moody. A., Spence, S., Orford, J., Volberg, R., Jotangia, D., Griffiths, M. D., Hussey, D. \& Dobbie, F. (2011). British Gambling Prevalence Survey 2010. London: The Stationery Office.

Whiteside, S. P., \& Lynam, D. R. (2003). Understanding the role of impulsivity and externalizing psychopathology in alcohol abuse: application of the UPPS impulsive behavior scale. Experimental and Clinical Psychopharmacology, 11, 210-?? Page numbers missing

Whiteside, S. P., Lynam, D. R., Miller, J. D., \& Reynolds, S. K. (2005). Validation of the UPPS impulsive behaviour scale: a four-factor model of impulsivity. European Journal of Personality, 19, 559-574.

Wiehler, A., \& Peters, J. (2015). Reward-based decision making in pathological gambling: the roles of risk and delay. Neuroscience Research, 90, 3-14.

Wilde, B., Goudriaan, A., Sabbe, B., Hulstijn, W., \& Dom, G. (2013). Relapse in pathological gamblers: a pilot study on the predictive value of different impulsivity measures. Journal of Behavioral Addictions, 2, 23-30.

Wray, I., \& Dickerson, M. G. (1981). Cessation of high frequency gambling and 'withdrawal'symptoms. British Journal of Addiction, 76, 401-405. 
Table 1. Means and standard deviations on the self-report measures for healthy controls (HC; $\mathrm{n}=54)$, and pathological gamblers (PG; $\mathrm{n}=54)$

\begin{tabular}{lccccccc}
\hline \multicolumn{7}{c}{ HC $(\boldsymbol{N}=\mathbf{5 4})$} & \multicolumn{2}{c}{ PG $(\boldsymbol{N}=\mathbf{5 4})$} \\
\hline BIS-IA & $\mathbf{M}$ & $\mathbf{S D}$ & $\mathbf{M}$ & $\mathbf{S D}$ & $\boldsymbol{F ( 1 , 1 0 6 )}$ & $\boldsymbol{p}<$ & $\boldsymbol{\eta}^{\mathbf{2}}$ \\
BIS-IM & 14.11 & 3.21 & 17.61 & 4.46 & 21.85 & .001 & .17 \\
BIS-INP & 18.70 & 4.04 & 23.50 & 5.51 & 26.58 & .001 & .20 \\
BIS-11 & 25.69 & 4.88 & 29.20 & 5.20 & 13.14 & .001 & .11 \\
CFC-I & 58.50 & 10.35 & 70.31 & 13.25 & 26.66 & .001 & .20 \\
CFC-F & 22.99 & 7.44 & 27.43 & 9.79 & 7.23 & .01 & .06 \\
CFC-14 & 30.46 & 8.23 & 27.83 & 10.17 & 2.18 & $\mathrm{~ns}$ & \\
AVERAGE k & 0.53 & 0.80 & 4.03 & 1.05 & 8.08 & .01 & .07 \\
BART & 23.44 & 17.55 & 42.59 & 25.28 & 20.91 & .001 & .16 \\
\hline
\end{tabular}

BIS-IA: Attentional Impulsiveness scale of BIS-11; BIS-IM: Motor Impulsiveness scale of BIS-11; BIS-INP: Nonplanning Impulsiveness scale of BIS-11; BIS-11: Barratt Impulsiveness Scale Total Score; CFC-I: Immediate scale of CFC-14; CFC-F: Future scale of CFC-14; CFC-14:Immediate scale of Consideration of future consequence Total scores; AVERAGE k: Average k value of the Monetary Choice Questionnaire; BART: Average number of pumps of unexploded balloons in the Balloon Analogue Risk Task. 
Table 2. Pearson correlation coefficients of measures among pathological gamblers $(n=54)$ and healthy controls $(n=54)$

\begin{tabular}{|c|c|c|c|c|c|c|c|c|c|c|c|c|}
\hline & 1 & 2 & 3 & 4 & 5 & 6 & 7 & 8 & 9 & 10 & 11 & 12 \\
\hline 1.SOGS & - & & & & & & & & & & & \\
\hline 2.BIS-IA & $.46^{* * *}$ & - & & & & & & & & & & \\
\hline 3.BIS-IM & $.48^{* * *}$ & $.74 * *$ & - & & & & & & & & & \\
\hline 4.BIS-INP & $.39 * *$ & $.60 * *$ & $.68 * *$ & - & & & & & & & & \\
\hline 5.BIS-11 & $.50^{* *}$ & $.86^{* * *}$ & $.92 * *$ & $.87 * *$ & - & & & & & & & \\
\hline 6.CFC-I & $.25^{* *}$ & $.47 * *$ & $.47 * *$ & $.47 * *$ & $.53^{* *}$ & - & & & & & & \\
\hline 7.CFC-F & -.13 & $-.28 * *$ & $-.38 * *$ & $-.40 * *$ & $-.41 * *$ & -.09 & - & & & & & \\
\hline 8.CFC-14 & $-.26 * *$ & $-.51 * *$ & $-.58 * *$ & $-.59 * *$ & $-.64 * *$ & $-.72 * *$ & $.75^{* *}$ & - & & & & \\
\hline 9.Small k & $.20 *$ & .09 & .16 & .14 & .15 & .13 & -.03 & -.10 & - & & & \\
\hline 10.Medium k & $.23^{*}$ & .07 & $.19 *$ & $.21 *$ & .18 & .08 & -.14 & -.15 & $.82 * *$ & - & & \\
\hline 11.Large k & $.19 *$ & .08 & .17 & .12 & .14 & .03 & -.11 & -.10 & $.79 * *$ & $.84 * *$ & - & \\
\hline 12. Overall k & $.21^{*}$ & .07 & .17 & .14 & .15 & .05 & -.11 & -.11 & $.89 * *$ & $.95^{* *}$ & $.95^{* *}$ & - \\
\hline 13.BART & $.40 * *$ & $.50 * *$ & $.46 * *$ & $.44 * *$ & $.52 * *$ & $-.32 * *$ & $-.32 * *$ & $.43 * *$ & .12 & .15 & .14 & .15 \\
\hline
\end{tabular}

Note. SOGS: South Oaks Gambling Screen; BIS-IA: Attentional Impulsiveness scale of BIS-11; BIS-IM: Motor Impulsiveness scale of BIS-11; BIS-INP: Nonplanning Impulsiveness scale of BIS-11; BIS-11: Barratt Impulsiveness Scale Total Score; CFC-I: Immediate scale of CFC-14; CFC-F: Future scale of CFC-14; CFC14:Immediate scale of Consideration of future consequence Total scores; Small k, Medium k, Large k: Small, medium and large hyperbolic discounting functions of Monetary Choice Questionnaire; Overall k: Mean k values of Monetary Choice Questionnaire; BART: Balloon Analogue Risk Task

$*=\mathrm{p}<.05, * *=\mathrm{p}<.01$ 
Table 3. Multivariate prediction of severity of gambling problems with a logistic regression model

\begin{tabular}{|l|c|c|c|c|}
\hline Predict & Beta & S.E. & $\begin{array}{c}\text { Wald } \\
\text { statistic }\end{array}$ & $\begin{array}{c}p \\
\text { value }\end{array}$ \\
\hline Step 1 & & & & \\
\hline BIS-11 & 0.084 & 0.020 & 18.01 & 0.001 \\
\hline Step 2 & & & & \\
\hline BIS-11 & 0.064 & 0.022 & 8.63 & 0.003 \\
\hline BART & 0.026 & 0.012 & 5.03 & 0.025 \\
\hline
\end{tabular}

Note. BIS-11= Barratt Impulsiveness Scale; BART= Balloon Analogue Risk Task 


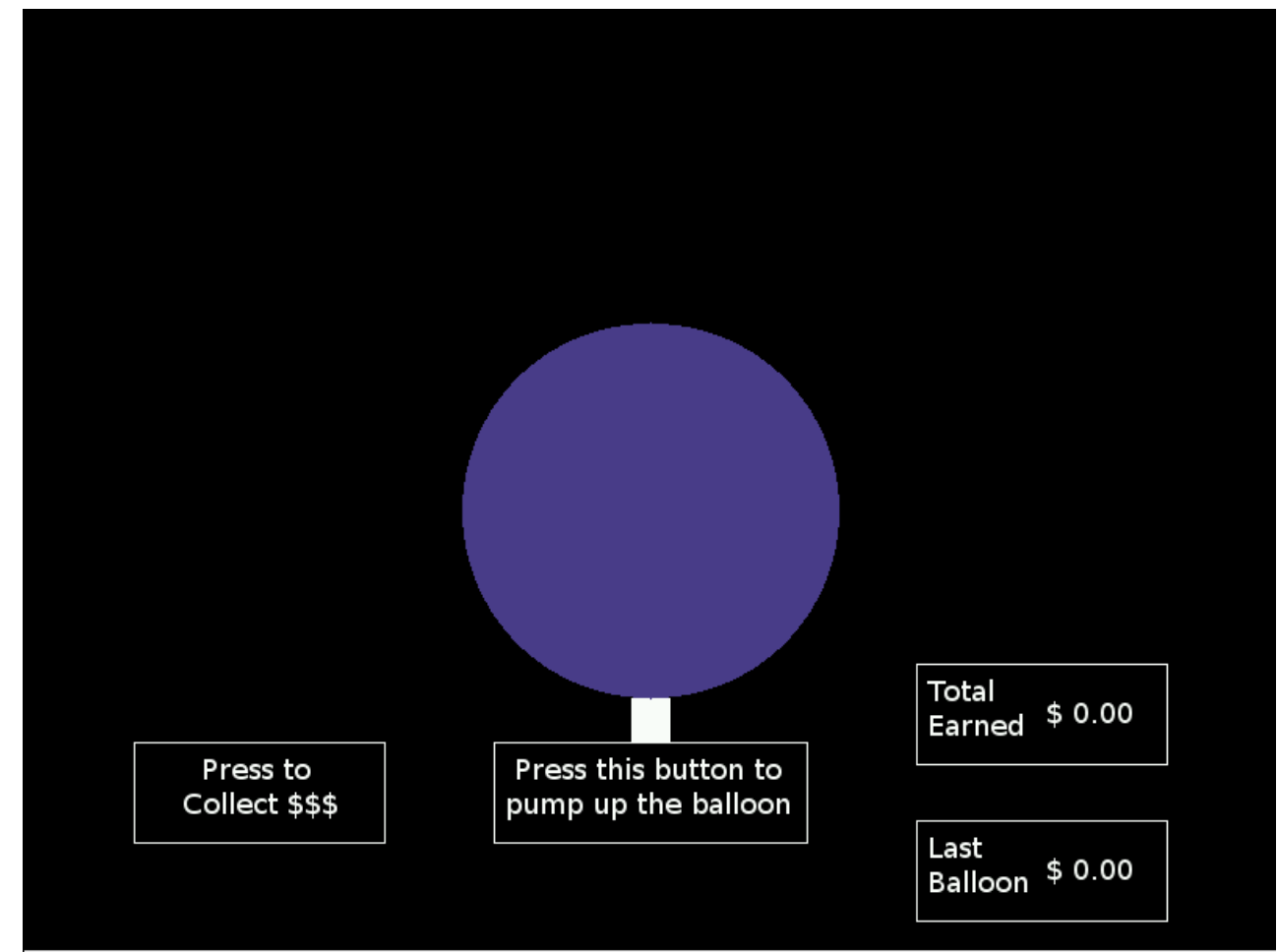

Figure 1. The Balloon Analogue Risk Task (Lejuez et al., 2002) 


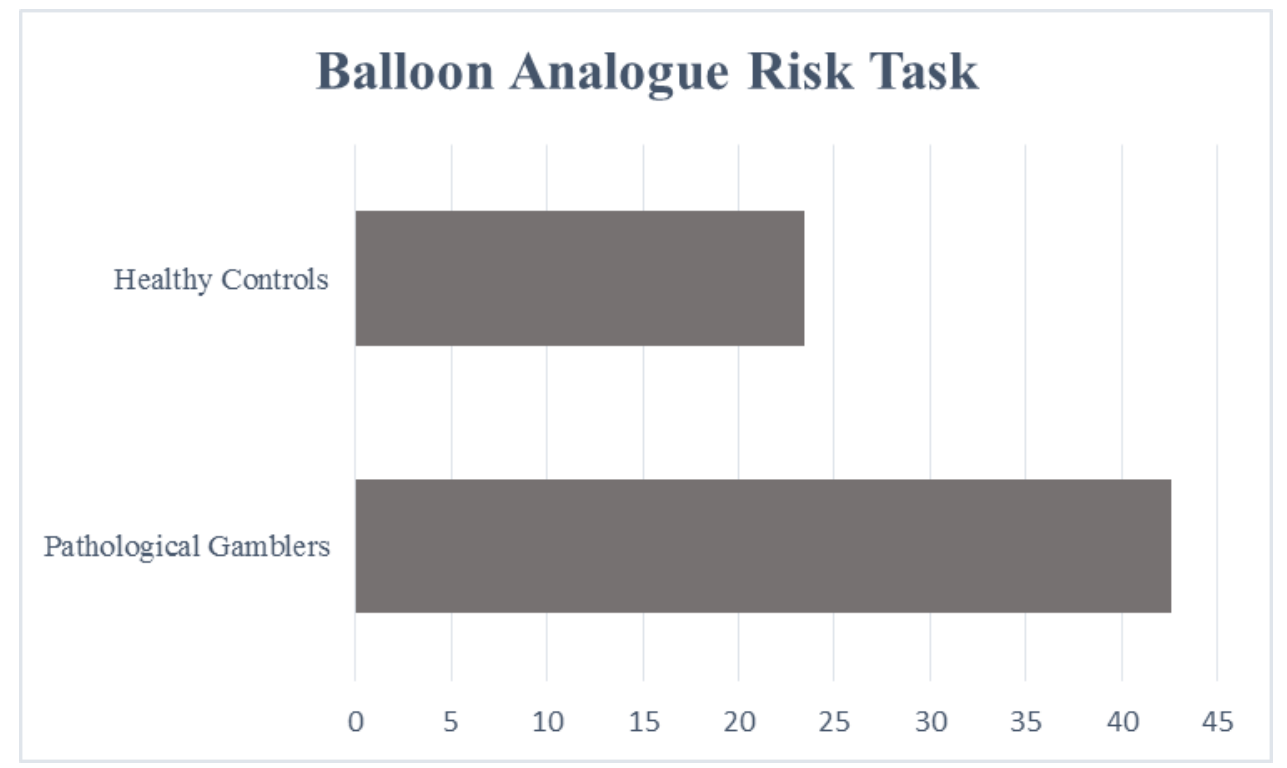

Figure 2. Mean number of pumps of the unexploded balloons in the BART

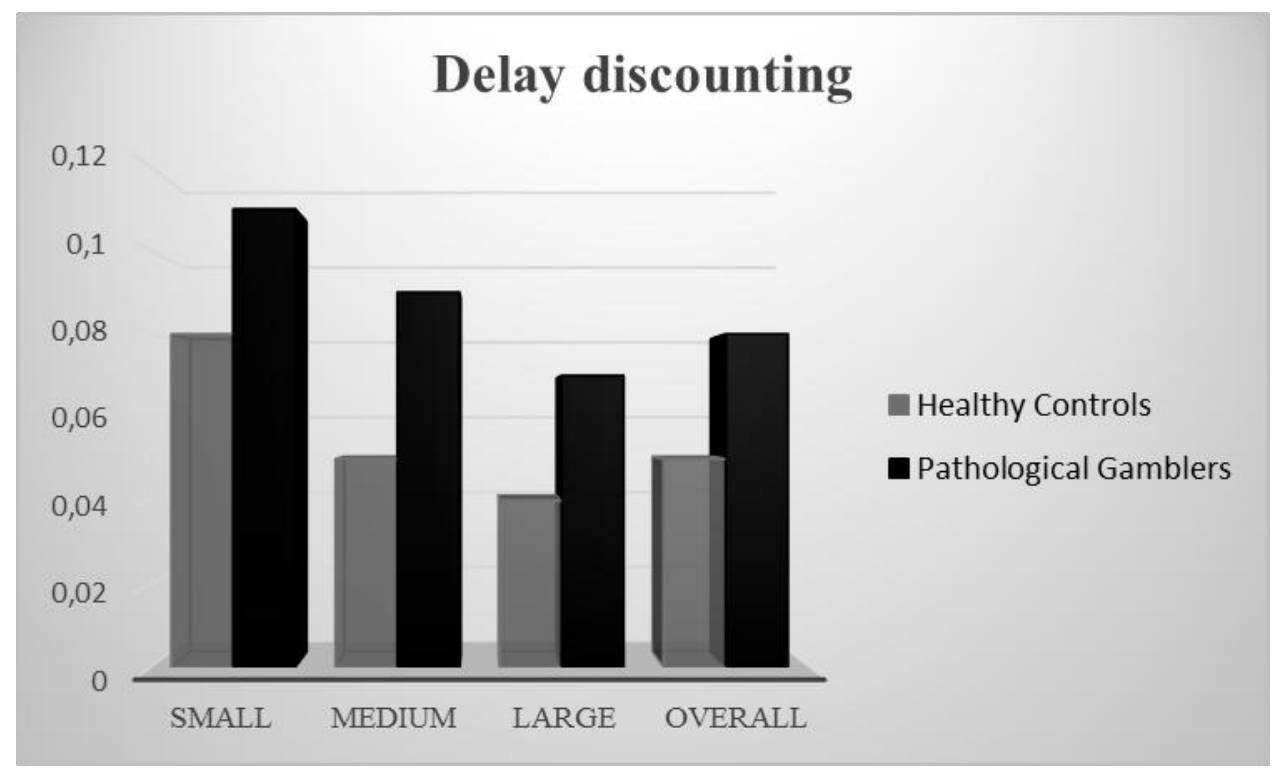

Figure 3. Delay discounting parameters for pathological gamblers $(\mathrm{n}=54)$ and healthy controls $(n=54)$. Data are separated into the three different reward amounts and average $\mathrm{k}$ value. 
